\title{
Improving Science and Mathematics Education with Computational Modelling in Interactive Engagement Environments
}

\author{
Rui Gomes Neves and Vítor Duarte Teodoro ${ }^{1}$ \\ Unidade de Investigação Educação e Desenvolvimento (UIED), Departamento de Ciências Sociais Aplicadas \\ (DCSA), Faculdade de Ciências e Tecnologia, Universidade Nova de Lisboa (FCT/UNL), Portugal
}

\begin{abstract}
.
A teaching approach aiming at an epistemologically balanced integration of computational modelling in science and mathematics education is presented. The approach is based on interactive engagement learning activities built around computational modelling experiments that span the range of different kinds of modelling from explorative to expressive modelling. The activities are designed to make a progressive introduction to scientific computation without requiring prior development of a working knowledge of programming, generate and foster the resolution of cognitive conflicts in the understanding of scientific and mathematical concepts and promote performative competency in the manipulation of different and complementary representations of mathematical models. The activities are supported by interactive PDF documents which explain the fundamental concepts, methods and reasoning processes using text, images and embedded movies, and include free space for multimedia enriched student modelling reports and teacher feedback. To illustrate, an example from physics implemented in the Modellus environment and tested in undergraduate university general physics and biophysics courses is discussed.
\end{abstract}

Keywords: Science and mathematics education; Learning and teaching; Interactive engagement environments; computational modelling PACS: 01.40.-d; 01.40.Fk; 01.40.gb; 01.40.Ha; 01.50.-i; 01.50.H-; 01.50.ht

\section{INTRODUCTION}

Science, mathematics and technology are deeply interconnected evolving structures of knowledge. On one hand, science is based on hypotheses and models, leading to theories, which have a strong mathematical character as scientific reasoning, concepts and laws are represented by mathematical reasoning, entities and relations. On the other hand, scientific explanations and predictions must be consistent with the results of systematic and reliable experiments, which depend on technological developments as much as these depend on the progress of science and mathematics.

For the modern professional communities in the diverse areas of science, mathematics and technology there is no doubt that such structures of knowledge develop as a consequence of individual and collective actions where modelling processes balance different elements from theory, scientific computation and experimentation. Science and mathematics education curricula and learning environments should then be based on pedagogical methodologies inspired in the professional modelling processes, defining specific context dependent strategies able to lead students through the different cognitive phases of the various professional modelling processes. This is an expectation that has been accumulating an increasing amount of scientific evidence, as many research efforts have been able to show that learning and teaching processes can effectively be enhanced when students work in environments with activities that approximately recreate the cognitive involvement of professional modelling activities [1]-[4].

The implementation of this kind of pedagogical methodologies requires the creation of learning environments that support meaningful learning paths [5] through the different phases of the modelling cycle, namely, qualitative contextual description, definition, exploration, interpretation and validation of mathematical models, communication of results and generalizations. On the other hand, since computational knowledge and technologies play a key role in the modern professional modelling processes, achieving an early epistemologically balanced integration of modelling activities that make an ample use of computational knowledge and technologies is fundamental. The teaching approach we propose to implement in this context involves the integration in the learning processes of interactive engagement activities structured around computational modelling experiments that span the range of different kinds of modelling

\footnotetext{
${ }^{1}$ Email addresses: rgn@ fct.unl.pt (corresponding author) and vdt@fct.unl.pt
} 
from explorative to expressive modelling [6]-[8].

\section{TEACHING APPROACH}

In our approach we consider that science and mathematics courses can be organized in a continuum of 4 complementary and interconnected components, namely, lectures where the theoretical foundations and first modelling examples are introduced, paper and pencil problem-solving lessons, computational modelling classes and experimental laboratories. To create an interactive engagement environment across all components, students are organized in collaborative group teams of two or three. During each class, the teams work on a set of interactive and exploratory learning activities which are structured around specific topics and aim to set up meaningful learning atmospheres approximately recreating the environments of professional modelling activities. Emphasis is placed on theoretical, experimental, computational and problem solving aspects in the corresponding course components, all appropriately articulated. Each new topical theme is introduced in a lecture class and afterwards the student teams are motivated to analyse, discuss and solve subsequent activities on their own using the theoretical, computational and experimental modelling guidelines provided by the lecture and associated documentation. Note that the teams are not left working alone but continuously helped during the exploration of the activities to ensure adequate working rhythm with appropriate qualitative and quantitative understanding. Whenever necessary, global class discussions are conducted to keep the pace, clarify doubts on concepts, reasoning or calculations that are common to several teams and for students work presentations.

All course activities are supported by PDF documents where the explanation of the fundamental concepts and methods, as well as the problem solving processes, is done using interactive text, images and embedded movies, with detailed sequential reasoning descriptions. Assessment activities involve modelling problems with instructions having various challenging levels of incompleteness. To increase the level of interactivity and promote digital communication competences, the documents include free working space to insert multimedia enriched student modelling reports and teacher feedback. Note that these interactive PDF documents depend on each specific science and mathematics course and each specific content level. The design is modular, rooted on the epistemology of modelling, allowing integration in a wide range of similar courses at various levels, including teacher training courses.

The computational modelling activities are introduced in a way that is balanced with the theoretical and experimental activities, and are conceived to span the range of different kinds of modelling from explorative to expressive modelling $[9,10]$. Their integration can be based on professional programming languages $[11,12]$ or on educational languages $[13,14]$. However, this option requires that students start by developing a working knowledge of programming, a problem that also accurs with professional scientific computation systems like Mathematica or Matlab. To reduce such cognitive load, particularly heavy for introductory levels, and focus the learning activities on the concepts and methods of science and mathematics, several computer modelling systems can be used instead [6]-[8], [15]-[18]. In this context, Modellus is particularly useful because it allows explorative to expressive modelling involving the simultaneous manipulation and analysis of several different model representations, namely, tables, graphs and animations with interactive objects whose properties are defined in a visible and modifiable mathematical model [6]-[8].

Naturally, all computer software tools, from programming languages to exploratory simulations, have advantages and shortcomings which depend on the activity content level and, for similar content level, on the modelling cycle stage. In our approach we consider the complementary use of a set of different tools, for example, Modellus [6][8], EJS [16], Physlet simulations [15] or PhET simulations [17], Excel, Mathematica or Matlab, and Java [11] or Python [12]. The guiding principle is to make the computer an effective tool for explorative to expressive modelling integrated in meaningful learning environments reflecting the epistemologies of modern science and mathematics, while simultaneously avoiding cognitive overhead factors such as too much programming and specific software knowledge. Using the range of complementary functionalities of different computational tools the activities can be designed to make a progressive introduction to scientific computation without requiring prior development of a working knowledge of programming, generate and foster the resolution of cognitive conflicts in the understanding of scientific and mathematical concepts and promote performative competency in the manipulation of different and complementary representations of mathematical models.

\section{AN ILLUSTRATIVE EXAMPLE FROM PHYSICS}

Consider the motion of a parachutist taking into account the effect of air resistance. The starting point to construct a mathematical model is to acknowledge that the parachutist can be represented by a point particle, located in the centre 


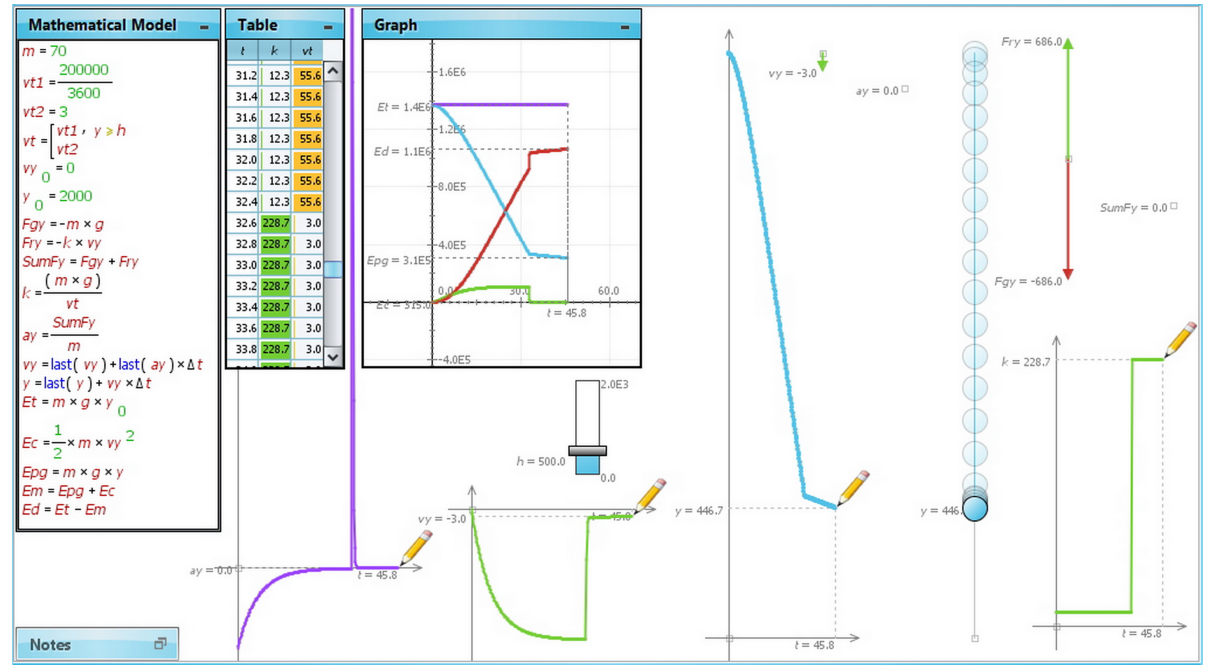

FIGURE 1. Using Modellus and Newton's equations written as Euler-Cromer iterations to model the motion of a parachutist taking into account the effect of air resistance. Before the opening of the parachute the air resistance coefficient $k$ is $12.3 \mathrm{Ns} / \mathrm{m}$ and after the opening of the parachute $k$ is $228.7 \mathrm{Ns} / \mathrm{m}$.

of mass, whose motion is governed by Newton's laws. Prior knowledge framing this problem involves knowledge about vectors, kinematics, constant acceleration applications of Newton's laws, comparing analytic, Euler and EulerCromer solutions [6,7], and knowledge obtained from observations of real parachute jumps. When air resistance is taken into account it is necessary to include in Newton's equations a force representing the action of the air on the motion of the particle. For many realistic situations, this force $\mathbf{F}_{r}$ points in the direction opposite to the velocity $\mathbf{v}$ and has a magnitude proportional to a power $\alpha$ of the speed $v, \mathbf{F}_{r}=-k v^{\alpha} \mathbf{u}_{v}$, where $k$ is the air resistance coefficient and $\mathbf{u}_{v}=\mathbf{v} / v$ is the unitary vector pointing in the direction of $\mathbf{v}$. Before the opening of the parachute, the sum of the gravitational force and the air resistance force has a magnitude that decreases with time and approaches zero when the magnitude of the air resistance force is equal to the weight of the parachutist. The speed reaches a constant value called the terminal speed given by $v_{t}=m g / k$, where $m$ is the mass of the parachutist and $g=9.8 \mathrm{~m} / \mathrm{s}^{2}$ is the acceleration of gravity. After this the parachutist keeps descending with a constant velocity (called terminal velocity). When the parachute opens $k$ increases and there is a new terminal velocity which is much smaller in magnitude.

For the majority of introductory level science and mathematics students solving Newton's differential equations with this force law is beyond the scope of their mathematical analysis capabilities. However, with computers and simple numerical methods like the Euler and Euler-Cromer methods, the mathematical modelling of the parachutist motion can be done at introductory level thus allowing a closer contact with the model referents. Student cognitive attention can then be focused on fundamental physical content leaving for a later consolidating stage the analysis of the more advanced mathematical physics structures. Here we illustrate how a computer modelling system like Modellus can be used with advantage in this context. A typical example considers a parachutist with mass $m=70 \mathrm{~kg}$ starting at a height $h=2 \mathrm{~km}$ (Fig. 1). Before the opening of the parachute the terminal velocity is $200 \mathrm{~km} / \mathrm{h}$ and after the opening of the parachute is $3 \mathrm{~m} / \mathrm{s}$. The mathematical model defines the applied forces, the gravitational force and the air resistance force as well as their net sum. Newton's equations of motion are integrated numerically with the Euler or Euler-Cromer methods. For simplicity, students can start with a vertical descent and take $\alpha=1$. The opening of the parachute can be done with a branching function or interactively manipulating $k$ taken as an independent variable. During the modelling activities students can explore the opening of the parachute at different heights, and interpret the graphs and tables of the components of the position vector, the velocity, the acceleration, the gravitational force, the air resistance force and their net sum as functions of time. Alongside these graphs and tables, the animation can display in real time the motion of a particle representing the parachutist, the stroboscopic trajectory, and the evolution of the vector diagrams with the velocity, the acceleration, the gravitational force, the air resistance force and the net force. The initial settings and the force law $\alpha$ parameter can be easily changed and the effect of such changes can be seen in the animation, graphs and tables, enhancing explorative and comparative modelling analysis. The model can also include the energy balance analysis based on the law of the conservation of energy (Fig. 1). 


\section{FIELD ACTIONS AND CONCLUSIONS}

The parachutist motion was one of the examples belonging to a series of interactive computational modelling activities created in the Modellus environment that we have tested during the implementation of our approach in the general physics and biophysics courses offered to the first cycle undergraduate university biomedical engineering and informatics engineering students at FCT/UNL. As evidenced by the content analysis of student coursework and evaluation tests, the series of computational modelling activities contributed successfully to identify and resolve many of the student difficulties in key concepts and processes in mathematics, physics and scientific computation. Two factors were central to achieve this: to have real-time on-screen correspondence between the animations with interactive objects and the object's mathematical properties defined in the model, and to have the opportunity of simultaneously manipulating several different representations. Students were able to create and explore models and animations, and not just act as simple browsers of computer simulations. In addition, students solved Newtonian models based on first order ordinary differential equations with constant and non-constant force laws, applying simple numerical methods, such as the Euler and Euler-Cromer methods, and understanding the conceptual and operational differences existing between numerical solutions and analytical solutions. Likert scale questionnaires showed that the majority of students considered favourably the interactive engagement group activities as well as the supporting PDF documents and software resources. Future action research will involve the development and testing of new interactive digital documentation and software resources for science and mathematics interactive engagement modelling activities with computational methods and tools, and will include further analysis of the corresponding learning and teaching processes.

\section{ACKNOWLEDGMENTS}

Work supported by Unidade de Investigação Educação e Desenvolvimento (UIED), Faculdade de Ciências e Tecnologia, Universidade Nova de Lisboa (FCT/UNL) and Fundação para a Ciência e a Tecnologia (FCT), Programa Compromisso com a Ciência, Ciência 2007.

\section{REFERENCES}

1. L. McDermott, and E. Redish, American Journal of Physics 67, 755-767 (1999).

2. J. Handelsman, D. Ebert-May, R. Beichner, P. Bruns, A. Chang, R. DeHaan, J. Gentile, S. Lauffer, J. Stewart, S. Tilghmen, and W. Wood, Science 304, 521-522 (2005).

3. W. Blum, P. Galbraith, H.-W. Henn, and M. Niss (Eds.), Modelling and Applications in Mathematics Education, Springer, New York, 2007.

4. D. Meltzer, and R. Thornton, American Journal of Physics 80, 478-496 (2012).

5. J. Mintzes, J. Wandersee, and J. Novak (Eds.), Teaching Science for Understanding: A Human Constructivist View, Elsevier Academic Press, Burlington, 2005.

6. R. Neves, and V. Teodoro, Journal Mathematical Modelling and Application 1, 2-15 (2010).

7. R. Neves, J. Silva, and V. Teodoro, Improving Learning in Science and Mathematics with Exploratory and Interactive Computational Modelling, in International Perspectives on the Teaching and Learning of Mathematical Modelling, Vol. 1, ICTMA14 - Trends in Teaching and Learning of Mathematical Modelling, edited by G. Kaiser, W. Blum, R. Borromeo-Ferri, and G. Stillman, Springer, Dordrecht, 2011, pp. 331-341.

8. V. Teodoro, and R. Neves, Computer Physics Communications 182, 8-10 (2011).

9. J. Bliss, and J. Ogborn, Journal of Computer Assisted Learning 5, 37-50 (1989).

10. J. Schwartz, Models, Simulations, and Exploratory Environments: A Tentative Taxonomy, in Foundations for the Future in Mathematics Education, edited by R. Lesh, E. Hamilton, and J. Kaput, Lawrence Erlbaum Associates, Mahwah, 2007, pp. 161-172.

11. H. Gould, J. Tobochnik, and W. Christian, An Introduction to Computer Simulation Methods: Applications to Physical Systems, Addison-Wesley, San Francisco, 2007.

12. R. Chabay, and B. Sherwood, American Journal of Physics 76, 307-313 (2008).

13. S. Papert, Mindstorms: Children, Computers and Powerful Ideas, Basic Books, New York, 1980.

14. A. diSessa, Changing Minds: Computers, Learning and Literacy, MIT Press, Cambridge, 2000.

15. W. Christian, and M. Belloni, Physlet Physics: Interactive Illustrations, Explorations, and Problems for Introductory Physics, Prentice Hall, Upper Saddle River, 2004.

16. W. Christian, and F. Esquembre, The Physics Teacher 45, 475-480 (2007).

17. C. Wieman, K. Perkins, and W. Adams, American Journal of Physics 76, 393-399 (2008).

18. A. Heck, E. Kadzierska, and T. Ellermeijer, Journal of Computers in Mathematics and Science Teaching 28, 147-161 (2009). 